\title{
Elección y temporalidad: el margen de la lectura
}

\author{
Choice and temporality: \\ the margin of reading
}

\section{Nora Bolis}

ReSUMEN:

En este artículo buscamos avanzar sobre algunas de las preguntas formuladas en el espacio de las Jornadas de Psicoanálisis de la Facultad de Psicología de la UNR, acerca de las dificultades en la lectura de la obra de Freud en los inicios del recorrido universitario en la carrera de Psicología. Este ámbito se ofrece a los docentes con el fin de dar a conocer el modo en el que pensamos nuestras prácticas. Algo de este "pensar" parece escurrirse, en el diario trajín de la enseñanza universitaria. Cotidianeidad ajetreada y a veces arrasada por una multiplicidad de fuerzas, conflictos o situaciones que nos llevan a un "duro esforzar" para encontrar la oportunidad del diálogo, del acercamiento a las preguntas y las lecturas de los estudiantes. Sin embargo, a veces esos encuentros se producen en el margen que se sustrae al tiempo difuso o enloquecedor en el que tropieza la vida institucional. Allí, en el ahuecamiento de un devenir pleno de sentidos cristalizados, se instaura provisoriamente otra temporalidad, donde la palabra revela su potencia evocadora, y ciertos anudamientos, nuevos, se enlazan a otros que "ya estaban".

\section{SUMMARY:}

In this article we seek to advance on some of the questions formulated in the space of the Psychoanalysis Conference of the UNR's School of Psychology, about the difficulties in reading Freud's work at the beginning of the university course. This field is offered to teachers to make known the way in which we think about our practices. Something of this "thinking" seems to slip away, in the day-to-day work of university teaching. Busy daily life and sometimes devastated by a multiplicity of forces, conflicts or situations that lead us to a "hard work" to find the opportunity for dialogue, the approach to questions and the readings of students. However, sometimes these encounters take place in the margin that subtracts from the diffuse or maddening rhythm in which institutional life stumbles. There, in the hollowing of a becoming full of crystallized senses, temporarily establishes another temporality, where the word reveals its evocative power, and certain knots, new, are linked to others that "already were". What would this "other time" be about, that can take place, from time to time in a class space 
¿De qué se trataría este "otro tiempo", que en un espacio de clases en la universidad puede de vez en cuando cobrar dimensión? ¿A qué operación nos remite y de qué modo se enraíza en la práctica clínica, sin que esta tenga lugar como ilustración o demostración? Trataremos de hilvanar una articulación entre el intervalo temporal, que se tensa en el hallazgo de lo nuevo como indicio lo que "ya estaba" sin tener lugar, y la operación que Lacan define como separación en el proceso de constitución subjetiva. Separación que precipita lo imposible en que se soporta el deseo en el hacer analítico, desplegando sus efectos, azarosamente, en el acto de lectura...en la Universidad.

\section{Palabras Clave:}

Lectura - intervalo - Alienación Elección - Temporalidad in the university? To which operation does it refer us and in what way is it rooted in clinical practice, without this taking place as an illustration or demonstration? We will try to weave an articulation between the temporal interval, which is tensed in the finding of the new as an indication of what "was already" without taking place, and the operation that Lacan defines as separation in the process of subjective constitution. Separation that precipitates the impossible in which desire is supported in analytic doing, deploying its effects, randomly, in the act of reading ... in the University.

\section{WORD PAD:}

Reading - Interval - Alienation Choice - Temporality 
LA LECTURA COMO VACIAMIENTO

DEL SABER

Nuestra práctica docente, nos ubica en un momento de la enseñanza, que podríamos designar como "Lectura de Freud en los inicios", dado que se trata de las primeras lecturas de textos de Freud por parte de los estudiantes. A su vez el programa de la cátedra Psicoanálisis 1 presenta un recorrido que comienza en las conceptualizaciones inaugurales de Freud acerca del funcionamiento del aparato psíquico. El trabajo que proponemos como "lectura" de Freud, se direcciona en la producción de vacíos, silencios, ahuecamientos sobre un saber que se completa a cada paso. Cierre que es efecto de la pregnancia del sentido común, encabalgado en nociones psicológicas y en especial en un código fragmentario de términos psicoanalíticos. Tal vez este último retazo de saber constituido en rumor resulte ser el más consistente, aquello que se escucha y circula señalizando una pertenencia difusa. Discurso que traza los bordes de una posible inclusión en el espacio universitario. ¿Qué consistencia toman esos saberes, al momento de encontrarse ante la letra freudiana? ¿Cómo situar la dificultad en textos inacabados, plagados de hipótesis contradictorias, de paradojas temporales no explicitadas como tales? ¿Cómo posicionarse ante la textura de lo imposible que hilvana esos textos?

A propósito de la posición de Lacan como lector de Freud e interrogando a su vez, nuestra posición como lectores de la obra de Freud y de Lacan, Claudio Glasman postula en "El Nombre-del-Padre: Un punto de partida":
"Con las obras de Freud y de Lacan tenemos al menos dos opciones: o somos sus creyentes servidores y las convertimos en monumentos del saber sin fisuras de cuya adoración y autoridad vivimos parasitándolas, para citándolas vivir de ellas o en cambio nos servimos de ellas y las hacemos redes vacías para poder atrapar el deseo en las letras singulares de los análisis que practicamos. También en los modos que reinventamos para transmitir la experiencia del análisis."(Glasman, C. 2006, p.26)

Si la lectura de la obra de Freud y Lacan las dispone al modo de redes con agujeros que operan como referencias posibilitantes en la escucha del decir de los analizantes, ¿cómo extender el entramado de estas redes vacías, agujereadas, en el campo de la enseñanza en la universidad? Los agujeros que anudan ese tejido, ¿se tornan grietas por donde cae toda posibilidad de interrogación? ¿Deberían recubrirse apresuradamente, al modo de los "pensamientos argamasa” del relato del sueño, otorgando coherencia a lo inaudito, a lo inasible? De no ser así, de insistir en su dificultad de cierre, ¿qué incoercible incomodidad alterará el progreso de la enseñanza en la universidad? Alteración de un devenir temporal progrediente de sumatorias y concatenaciones en la construcción de los conceptos, por un despliegue en zig-zag de avances, retrocesos y detenciones. Detenciones que pueden conjeturarse como aplazamientos necesarios de un cierre anticipado.

En el artículo mencionado Glasman sitúa puentes inter-textuales, entre un señalamiento repetido por Freud en el hallazgo de la interpretación en el Hombre de los lobos y lo que Lacan define como 
punto almohadillado en el Seminario 3 (1956) sobre "Las Psicosis". Puentes tendidos por la repetición de términos como "convergencia, todo se irradia, hilos, lineas, retroactivamente” (p.28). Esto lo lleva a afirmar que estas "claves" de lectura permiten encontrar un movimiento de Freud a Lacan, en la lectura que realiza Lacan de Freud, como relación-separación con el Otro.

"Se trata de la singularidad de una lectura, de la puesta en acto del método analítico, y de cierta concepción de lo que es la estructura del sujeto que por estas vías irrepetibles de la repetición, se constituye, se co-instituye en su relación-separación con el Otro.” (Glasman, 2006, p.28-29)

¿Qué nos dice Freud sobre el modo de construir los conceptos en el movimiento de aproximación vacilante a aquello que sale al encuentro en la experiencia? En el texto publicado en 1915, "Pulsiones y sus destinos", advierte sobre la rigurosa dificultad de su apuesta, cuanto más logra aproximarse a aquello que quiere volver inteligible, se abre como abismo inevitable, un margen de indeterminación, entre el esfuerzo de la razón y la verdad esquiva que anida en la exploración clínica:

"Y más insoslayables todavía son esas ideas -los posteriores conceptos básicos de la ciencia- en el ulterior tratamiento del material. $\mathrm{Al}$ principio deben comportar cierto grado de indeterminación; no puede ceñirse con claridad su contenido. Mientras se encuentran en ese estado, tenemos que ponernos de acuerdo acerca de su contenido por la remisión repetida al material empírico del que parecen extraídas, pero que, en realidad, les es sometido. En rigor poseen entonces el ca- rácter de convenciones no obstante lo cual es de interés extremo que no se las escoja al azar... Sólo después de haber explorado más a fondo el campo de fenómenos en cuestión, es posible aprehender con mayor exactitud sus conceptos básicos y afinarlos para que se vuelvan utilizables en un vasto ámbito" (Freud, 1915, p.113)

Siguiendo esta vía, podemos pergeñar la operación de la lectura como extracción de la diferencia, en el hallazgo de un punto de convergencia y repetición. Allí donde el sentido que anudan los conceptos es convención e indeterminación...al mismo tiempo.

Lacan en el Seminario de 1964, "Los cuatro conceptos del psicoanálisis”, transcribe la postulación freudiana que señalamos, desde la "aproximación" del concepto a cierta analogía con el cálculo infinitesimal: el límite.

\footnotetext{
"Nuestra concepción del concepto entraña que este se establece siempre mediante una aproximación que no carece de relaciones con la forma que impone el cálculo infinitesimal. En efecto si el concepto se modela según un acercamiento a la realidad que él está hecho para aprehender, solo mediante un salto, un paso al límite, cobra forma acabada realizándose.” ( Lacan, 1964, p.27)
}

Atendiendo a la sugerencia de lectura propuesta por Glasman, encontramos que en el intento de precisar la especificidad psicoanalítica del trabajo de conceptualización Freud y luego Lacan interponen el término aprehender, entre concepto y realidad empírica en Freud, entre concepto y la "realidad" en Lacan. Este término intenta precisar la relación inacabada, del concepto a los fenómenos, relación en 
movimiento dice Freud, en la introducción de Pulsiones y sus destinos, donde nos advierte que el "progreso del conocimiento no tolera rigidez alguna, tampoco en las definiciones" (Freud, 1915, p.113).

¿Podemos suponer que la acción de aprehender que señalan Freud y Lacan como modo de operar con los conceptos en la clínica, se desliza como puntuación necesaria en el discurrir de la enseñanza? La noción de aprehender es enlazada por Lacan con el "salto" como aquello que realiza el concepto. Desde esta perspectiva es posible vislumbrar que en determinado momento del diálogo iniciado alrededor de la lectura de los textos freudianos, ese movimiento entre la captura y la pérdida se produce como vacío, efecto de la interrogación, que bordean insistentemente las formulaciones freudianas. El salto al límite que Lacan sugiere, es un movimiento asintótico, que refuerza lo imposible de la adecuación, que empuja a la realización como inacabada. Quiero decir, es esa huella del inacabamiento que horada el concepto, la que empuja a nuevas elaboraciones. Elaboraciones donde lo nuevo transcribe lo que ya estaba atópicamente, sin tener lugar, en otro espacio, que se recorta a partir de la inscripción. Por lo cual podríamos inferir que lo nuevo no es el concepto en sí mismo, sino el espacio en el que se reescribe cada vez. La hoja, el mapa de hilos elaborativos que anudan aquí y allá hallazgos en el recorrido propuesto por el texto a descifrar.

Lo asintótico como movimiento inherente a la realización del concepto indica la existencia de una hiancia insalvable. Esta hiancia es eficaz, opera en los distintos campos del hacer del psicoanálisis: la enseñanza, la lectura, el análisis. Produce en esos campos la necesariedad de un intervalo, que se abre entre la pregunta y el hallazgo. La pregunta, al formularse esboza la dimensión de la elección y el hallazgo como anudamiento provisorio deviene efecto de la separación. Separación que atañe al campo del saber, como discurso que presenta su punto inconsistencia en el desvanecimiento que enlaza al sujeto. El intervalo que se abre entre ambos momentos puede pensarse como alojamiento "temporal" del sujeto, en tanto su acto hilvana a posteriori, en discontinuidad, los hilos que entretejieron la elección. Juan Ritvo (2002) en las notas sobre "Acto, decisión, alienación" publicadas en Revista Conjetural N ${ }^{\circ} 40$, desprende la noción de salto de su referencia matemática al articularla al acto. Subraya la discontinuidad en la que se sostiene el acto y el establecimiento paradojal de una "extraña" continuidad a posteriori:

"Es preciso saltar porque la conclusión no se desprende espontáneamente de las premisas; Es preciso saltar porque entre razón y razón hay intersticios incolmables, que solo el razonamiento matemático puede colmar porque es puramente formal, es preciso saltar porque entre el hecho y el valor, hay discontinuidad....Sin embargo, el salto establece, en el vértigo de la inexistencia del Otro, una extraña continuidad a posteriori, tal y como opera en el fin de análisis...." (Ritvo, 2002, p.30)

En el diálogo que puede desplegarse en algunos momentos del discurrir de la enseñanza universitaria, la "orientación" que bosqueja el docente sitúa, mojona al modo de un relevamiento topográfico, los agujeros, las inconsistencias, los vacíos en el texto. Texto que por presentar estos agujeros, produce una fuerte "demanda" 
de explicación, de ilustración, de integración a lo ya sabido. Sin embargo, el detenimiento y desasosiego ante la emergencia de dichos vacíos, puede desencadenar a posteriori en un segundo tiempo de la lectura, enlaces desconocidos hasta ese momento. Puntualizar estos momentos, instala un compás de espera...inesperado. Habilitar silencios, esboza al modo de una pulsación sin compás fijo, el margen de la lectura. Las aproximaciones, las conjeturas, los balbuceos que arriesgan una pregunta, van configurando las tramas de una lectura. Urdimbres que mojonan una temporalidad a advenir. Efecto de la separación que se pone en acto al situar como puntuación, la dificultad de cierre en un saber totalizante. Separación que no diferencia de modo tajante dos campos en juego, sino que los anuda alrededor de la imposibilidad que instaura el discurso del psicoanálisis.

En diálogo con el artículo de Ritvo en la mencionada revista, Juan Molina reconfigura la noción de dualismo, o dualidad que esboza Lacan alrededor de la elección. Molina se pregunta, ¿la alienación plantea una alternativa entre dos conjuntos disjuntos?

"Pero lo que especifica esta presentación de los términos de la alternativa alienatoria, no es tanto que los mismos no sean disjuntos, sino que su intersección no contiene más que un vacío"....”Digamos mejor que aquí es donde tenemos que despegarnos del artificio matemático, puesto que en Lacan se hace jugar esa intersección como un punto de articulación. De esa lúnula que no contiene ningún elemento contable en uno de los conjuntos, está hecho el anudamiento del sujeto con el lenguaje; allí tendríamos que situar la estructura de la pulsión" (Molina, 2002, p.41)

El margen como espaciamiento que se extiende de cuando en cuando, allí donde la lectura entreteje la enunciación de una pregunta, instaura un intervalo temporal, en la tensión entre un enigma a descubrir y un corte en los desplazamientos de sentido. Este movimiento que intentamos ubicar como un momento particular de la enseñanza donde la lectura se inicia, puede pensarse como salto, en tanto enlaza sin borrar la discontinuidad, campos de saber heterogéneos. ¿Por qué la apertura de esta temporalidad involucra una elección? ¿De qué modo puede pensarse la elección en este marco que recorta un espacio de enseñanza en la universidad? $¿$ Es conjeturable que a cada paso, en cada vacilación que se acuña en la lectura, algo de la elección se pone en juego?

\section{ELECCIÓN, TEMPORALIDAD, EMERGENCIA DE LA ALTERIDAD}

Elección y sujeto son conceptos indisociables, desde la constitución subjetiva y sus operaciones fundantes. En función de aproximarnos al concepto desde un sesgo que bordee la noción de temporalidad, trazamos un breve recorrido por el escrito de Søren Kierkegaard publicado en 1843, "O lo uno o lo otro". En este texto escrito al modo de un diálogo en el que se interroga a un joven, el juego de oposición entre la posición ética y la vida estética se despliega como representación de la encrucijada que conlleva la elección como determinación del sujeto. La escritura dramática de este filósofo, expone de 
un modo luminoso los hilos que tensan la elección como encrucijada vital. Estos hilos permiten una aproximación a la noción de elección forzada en el Seminario Los cuatro conceptos fundamentales del psicoanálisis (Lacan, 1964), desde una argumentación que modela la temporalidad que se instaura en la elección.

La elección tal como la va delineando Kierkegaard, no se trata de la decisión entre posibilidades que se sopesan o valoran, sino del yo enfrentado al acto de elegir. Para Kierkegaard la ética supone la elección mientras que la estética conlleva la indiferencia ante la elección. A su vez estas dimensiones configuran temporalidades diferentes. Mientras que la estética conlleva la inmediatez, lo pasajero y disperso, la ética configura un devenir que articula de un modo particular, el pasado al presente orientando un futuro posible. ¿Qué elige el sujeto? Al yo devenido en una historia, singular y social. La vida estética conlleva la multiplicidad de las inclinaciones y la discontinuidad temporal representada por lo efímero, lo que sucede sin encadenarse, sin marca. La elección implica un salto cualitativo, una apuesta en la cual el sujeto se "asume" como dividido por la falta de garantías, por el vértigo de una creencia sin certezas. Elegirse a sí mismo encierra una contradicción o paradoja temporal: "Ese sí mismo no existía con anterioridad, puesto que llegó a ser en virtud de la elección, y sin embargo existía puesto que era él mismo" (p.196).

La elección conduce a una relación del sí mismo con su historia, pero sobre todo, con aquello que hereda. Una máxima identidad en algo que no es propio, y que debe conducir al arrepentimiento. La herencia, como historia presenta una necesaria exterioridad que debe ser asumida como "sí mismo". ¿Por qué el término asumir? Porque Kierkegaard diferencia la elección de sí mismo de la creación de sí mismo. Hay determinación y libertad absoluta en ese acto. Pero hay un acto psíquico que no se puede eludir y que conlleva una apuesta, en términos absolutos. Absoluto y eterno, si bien son términos que conducen a una dimensión religiosa, adquieren en este texto connotaciones de radicalidad y exterioridad.

El texto de Kierkegaard permite dimensionar este anudamiento, aun cuando su concepción de subjetividad, el "sí mismo", alude a una comunicación particular con Dios por la vía del arrepentimiento. Pero justamente esta vía del arrepentimiento como amor a Dios trasciende el aspecto religioso, abriendo a una interrogación filosófica sobre el estatuto del ser. Un ser que no puede ser captado en la mediación, que se perfila en una radical exterioridad constitutiva. Un sí mismo otro. Si bien el sí mismo lleva a Kierkegaard a la relación con Dios, pone en juego fundamentalmente un modo singular de relación con la Ley, entendiéndola como alteridad radical que debe ser asumida por la vía de la elección.

La elección como decisión entre alternativas que podrían analizarse desde una objetividad ajena a las determinaciones subjetivas, no es la elección desde el punto de vista ético. Ubicar estas alternativas en una objetalidad, como opciones entre esto o aquello, supone pensar a un yo por fuera del campo de determinaciones. Pero a su vez esa exterioridad en relación a lo que se elige genera la indiferencia propia de la vida estética. Delimita así dos tipos de exterioridad, en tanto una desafecta al sujeto y otra le concierne de modo íntimo. Kierkegaard ubica en ese campo, el de la indiferencia del joven el de la inconstancia 
y la inmediatez, a la melancolía. El desapego por las cosas del mundo es la melancolía basal en que transcurre la vida en su dimensión estética. Reprimir o postergar el movimiento que confronta a la elección lleva a la melancolía. La desesperación lleva a otra relación con la verdad del sí mismo, da cuenta de una proximidad a la verdad. Verdad que no se presentará como saber, sino como elección. Pero la desesperación no es la elección sino su momento lógicamente anterior. La desesperación parecería dar cuenta de la concreción que conlleva la elección ética, como verdad subjetiva. Esta desesperación interpela al yo y lo conduce a elegirse a sí mismo.

Elección que toma la dimensión de un acto: "Yo no me creo a mí mismo me elijo a mí mismo".(p.196) El sí mismo se plantea en una exclusión interna, respecto al yo y sus objetos. El sí mismo conlleva una división en el yo, que es efecto de una herencia inexplicable y en cierta medida inabordable. Acceder al sí mismo comporta una elección que antecede al individuo como condición de su genuina humanidad. El querer confrontarse a la elección, es la posibilidad de no perderse a sí mismo. Kierkegaard sostiene que se altera la elección cuando el hombre la posterga "por más que delibere y delibere creyendo mantener separadas las opciones. Si el hombre no ha elegido, otros eligieron por él, se pierde a sí mismo". (Kierkegaard, 1843, p.154)

En la elección eso que ya estaba se instaura como antecedente. La elección como alternativa ética, comporta una división subjetiva, en tanto aquello que se asume, revela la dimensión de alteridad constitutiva del sí mismo. Se constituye la dimensión de lo porvenir a partir del acto que da lugar a una alteridad constitutiva de la subjetividad, el sí mismo. La elección es parte de la alienación fundante de la subjetividad. Alienación que determina un campo de desconocimiento, de imposibilidad de saber que fuerza a la elección como salto cualitativo. En la medida en que el sujeto se deja atravesar por la elección, se configura una nueva dimensión temporal. El acto configura un tiempo a advenir.

Desde el psicoanálisis, es en función de la falta en el Otro, del vacío inherente al intervalo significante, que el sujeto se produce al modo de una apuesta y en el despliegue de nuevas significaciones, enlaces nuevos que a posteriori se anudan a algo que "ya estaba" sin tener existencia . En el campo de la lectura podríamos afirmar que no hay "experiencia de lectura" si en otro campo no se produce lo que Freud denomina un nuevo acto psíquico, como este enlace a posteriori, en transferencia, de lo que se anticipa como no realizado.

\section{AlienACiÓn, SEPARACIÓN, INVENCIÓN DEL INTERVALO}

La elección como acto psíquico que configura una nueva temporalidad es una operación que está en los fundamentos de la constitución subjetiva. Es en la lógica de la alienación primordial en que se constituye la subjetividad donde la elección cobra su determinación. Siguiendo la argumentación de Jacques Lacan sobre las operaciones fundantes del sujeto para el psicoanálisis, en "Los cuatro conceptos fundamentales del psicoanálisis", la elección es una operación necesaria, como efecto de la alienación primordial. Se plantea una elección imposible, dado que cada uno de los campos "posibles" para el 
alojamiento del sujeto, -el ser, el sentidoes un campo agujereado, cercenado.

El vel de la alienación se define por una elección cuyas propiedades dependen de que en la reunión uno de los elementos entrañe que sea cual fuera la elección, su consecuencia sea ni lo uno ni lo otro. (Lacan, 1964, p.219)

La alienación es una elección que plantea una imposibilidad, porque no es elección de esto o aquello. En el vel de Lacan, no hay posibilidad de elegir el ser sin que se pierda el sentido, sin que el sujeto "caiga” en el sin-sentido. Si elige el sentido, es un sentido cercenado por el sin-sentido que es marca del inconsciente. No hay posibilidad de ubicarse de modo pleno en ninguno de los dos campos. Cada uno de ellos va a estar agujereado por el punto de vacío, de hiancia que al mismo tiempo, los anuda en la alternancia propia de la repetición.

Lacan ubica esta hiancia en el intervalo que se abre entre el par de significantes primordiales, aquellos que están primordialmente reprimidos. Este par de significantes y el intervalo que los encadena, determinan el campo del Otro para el sujeto en el esquema de la alienación. Esta determinación produce el vel de la alienación y al mismo tiempo el factor letal constitutivo de esa instauración alienante. Ese intervalo, esa hiancia va a ser el lugar del posible alojamiento del sujeto. Posibilidad que anida una imposibilidad radical, ya que implica una elección entre campos no separables como opciones unitarias. Se trata de un destino que debe ser elegido, $\mathrm{y}$ en el momento que se elige, pierde su consistencia, hace su emergencia la libertad. La libertad sólo es pensable, teniendo en cuenta el sin sentido que anida en el campo del Otro, como lugar del senti- do. Este sin-sentido es el factor letal, que posibilita la emergencia del sujeto. Es en tanto cae, afánisis, tomado en su ser por la alienación al sentido, los sentidos provistos por el Otro, que el vacío, la falta inherente a ese campo será la condición de su emergencia. Es aquí donde Lacan ubica el sentido último de la interpretación en el análisis. Más allá de las significaciones, productos, retoños de lo reprimido primordial, la interpretación apuntará a ubicar el sin sentido que articula aquellos significantes determinantes en la historia del sujeto.

La separación es efecto del factor letal, la caída del sujeto, del ser del sujeto, es condición del encuentro con la falta. Lacan ubica la separación como la intersección entre los campos del ser y del sentido, intersección que no es más que un vacío. Este movimiento segundo implica una torsión, algo se inscribe pero en otro tiempo. Es decir que lo que aparece a partir de la separación, no es del mismo orden, o no se inscribe en la misma superficie. Dice Lacan que la relación del sujeto al Otro es de circularidad, pero en el retorno hay una torsión, un giro que desarma esa circularidad. Hay asimetría en esa circularidad. Esta asimetría en la circularidad de la relación del sujeto al Otro, se presentifica en que no hay una respuesta directa. Es decir, cuando el sujeto en ciernes se encuentra con la falta inherente al campo del Otro, responde con su propia falta. Una falta responde a otra falta, produciendo un "intervalo temporal" entre ambas. La segunda falta se suscita como respuesta a la primera, he allí el proceso de transmisión. Es a partir de situar el punto de vacío en el campo del Otro que la transmisión es posible. Es la pregunta del sujeto lo que sitúa a posteriori los efectos de la transmisión. 
Es porque no hay respuesta directa, por lo que el sujeto puede hacer experiencia, en tanto experiencia subjetiva. El vel de la alienación al provocar una elección, genera la tensión inaugural de un segundo momento, el del sujeto en ciernes. La alienación fundante genera una tensión inaugural en la conminación al sujeto a elegir. Esta tensión temporal inherente a la encrucijada que se presenta al sujeto produce temporalidades, como tiempos a advenir en la elaboración psíquica.

Puede conjeturarse que el trabajo de interrogación del saber que adviene del encuentro con los textos de Freud pone en juego la elección como operación que instaura la división subjetiva, desde el vacío que enhebra el saber. Es la tensión que inaugura esta elección, la que "provoca" un tiempo potencial de producción de diferencias en el hacer de la lectura. En este sentido puede pensarse la lectura como experiencia, dado que enlaza al sujeto al “conminarlo" en la elección. Basculación que fuerza a una precipitación donde el hallazgo no va a conformar una unificación del saber, sino una nueva tensión que prorroga y a su vez anticipa la elaboración de una nueva pregunta. Ese breve intervalo entre el aprisionamiento en lo ya conocido y el suspiro de incertidumbre que otorga la lectura, podría figurarse como el margen que la elección instaura en el trabajo de la lectura. Margen como efecto necesario de la alienación fundante que nos encadena al lenguaje y a la vez aleatorio en su emergencia, ya que no hay garantías en cuanto a las posibilidades azarosas de un diálogo por venir...

Ese margen incierto, latido del texto que captura el trabajo la lectura, puede elidirse o desmentirse repetidamente, en el intento de conjugar un desarrollo psico- sexual. La posibilidad de abrir un tiempo “otro" al despliegue progrediente de la enseñanza universitaria, arrastra su contingencia. Cada encuentro singular con la letra freudiana pone en juego la asimetría de un diálogo en suspenso. Incomodidad del desconocimiento allí donde se reinstala a cada paso la sutura del vacío ineludible en que se teje la lectura de los textos freudianos.

\section{REFERENCIAS BiBLIOGRÁFICAS}

Freud, S. [1914-15] (1979). Trabajos sobre metapsicología y otras obras. Obras Completas Tomo XIV . Buenos Aires: Amorrortu Editores.

LaCAN, J. [1964] (1987). Los cuatro conceptos fundamentales del psicoanálisis. Buenos Aires, Ediciones Paidos.

Glasman, C. (2006) "El Nombre-del-Padre: Un punto de partida", El Padre que no cesa. Buenos Aires, Letra Viva, 23-56.

Molina, J. (2002) “Argumentaciones del cogito freudiano." Revista Conjetural. Buenos Aires, Ediciones sitio, Vol.38, 33-44.

KierkegaARd, S. [1843] (2007). O lo uno o lo otro. Madrid: Editorial Trotta.

Ritvo, J. (2002) “Acto, decisión, alienación.” (Notas). Revista conjetural, Buenos Aires, Ediciones sitio Vol.38, 19-32. 
RESEÑA CURRICULAR DEL AUTOR

Nora Bolis es psicoanalista, Doctora en Psicología, UNR. Profesora en la cátedra de Psicoanálisis 1. Ha publicado diversos artículos sobre problemas conceptuales de la metapsicología freudiana e investigaciones en Psicoanálisis y Educación en Revistas especializadas. 